\title{
TOWARDS AN OPTIMIZATION OF THE LHC INTERSECTION REGION USING NEW MAGNET TECHNOLOGY*
}

\author{
P. McIntyre, A. Sattarov, Texas A\&M University, College Station, TX 77883 U.S.A. \\ J-P. Koutchouk, CERN, Geneva, Switzerland
}

\begin{abstract}
A strategy for optimizing the intersection region of LHC is presented. The quadrupole triplet is moved closer to the intersection point, within the footprint of the detectors. The $350 \mathrm{~T} / \mathrm{m}$ inner quadrupole is designed using a structured cable that incorporates internal refrigeration with supercritical helium. The separation dipole utilizes a levitated-pole design.

This approach opens the possibility to achieve a minimum $\beta^{*}$ without increase of the chromatic and geometric aberrations. The crossing angle can be reduced, and the aperture required in the quadrupole triplet is reduced significantly. The additional space that is opened in the lattice can be used for additional quads to improve the insertion matching.
\end{abstract}

\section{HIGH-LUMINOSITY DESIGN}

Each high-luminosity intersection region (IR) in the Large Hadron Collider LHC poses three significant challenges for the match of the physics mission of the collider with the optical train of the arcs: focal optics, crossing and separation of the two beams, and management of heat and radiation from secondary particles that are produced in proton-proton interactions and then lost into the cold mass of the magnets. The focal optics is optimized by placing the quadrupole triplet as close as possible to the interaction point (IP). This minimizes $\beta_{\max }$ and also minimizes the sensitivity to chromaticity and to alignment errors and error multipoles in the IR magnets. Preliminary discussions with both experimental teams indicate that the first quad could be placed at a distance $\mathrm{s} \sim 12 \mathrm{~m}$ from the IP.

The beams must be crossed at small angle and then separated, and the separation dipole must be placed as close to the IP as possible to minimize the number $\mathrm{N}_{\mathrm{s}}$ of subsidiary bunch-bunch crossings that produce long-range beam-beam interactions.

Finally the proton-proton interactions produce an intense flux of particles in the forward directions. Many of those particles strike the first quad $\mathrm{Q}_{1}$ and the separation dipole $\mathrm{D}_{1}$. In a close-in placement of $\mathrm{Q}_{1}$, a heat load of $\sim 300 \mathrm{~W}$ is estimated for the design beam intensity. The particles swept from the beam tube in $D_{1}$ will deposit $\sim 3$ $\mathrm{kW}$ in its side walls [1]. Those 'first wall' elements must be designed to minimize cryogenic heat load, maximize heat transport, and accommodate radiation damage.

*Work supported by US Dept. of Energy, grant \# DE-FG03-95ER40924, and by EC Research Infrastructure Activity FP6: 'Structuring. the European Research Area' program, CARE-RII3-CT-3003-506395.

\#p-mcintyre@physics.tamu.edu
Previous designs for the IR [2] have been based upon a separation of $21 \mathrm{~m}$ between the IP and the IR, and upon conventional designs for the superconducting quadrupoles and dipoles. In this paper a new approach to the magnet designs is presented, specifically chosen to address the above challenges. The IR is configured to take best advantage of the new approach, moving all elements as close as possible to the IP as shown in Figure 1. Table 1 gives the main parameters of the magnetic elements. The improved performance provided by the new designs is used to minimize $\beta^{*}, \beta_{\max }$, and $\mathrm{N}_{\mathrm{s}}$. Additional quads can then be placed in the space opened in the outer regions of the straight section to facilitate the matching of the IR to the arc lattice.

\section{QUADRUPOLE TRIPLET}

For the purposes of this design we examine the case that no shielding is placed in front of $Q_{1}$ so that it can be located as close as possible to the IP. $Q_{1}$ must then be designed as an ironless quadrupole because it is located within the field region of the magnetic elements of each detector. The heat and radiation load from particle losses within the coils places extreme requirements on heat transport within the superconducting coil and its structure. In particular it is unlikely that any vacuum-impregnated coil could maintain the limited temperature profile needed for superconducting operation.

We have developed a $\mathrm{Nb}_{3} \mathrm{Sn}$ quadrupole coil design that would employ to advantage a structured cable [3] containing 6 round strands of superconductor around a spring tube core. The cable was originally developed for use with Bi-2212 in order to provide direct contact of all strands with flowing liquid helium. A cross section of the cable is shown in Figure 2a.

The six strands are arranged in a 6-on-1 cable-inconduit configuration in which the center element is a thin-wall (40 $\mu \mathrm{m}$ wall) tube made of Inconel X-750. The center tube is spring-tempered and retains the temper through the $650 \mathrm{C}$ heat treatment of the $\mathrm{Nb}_{3} \mathrm{Sn}$ strands.

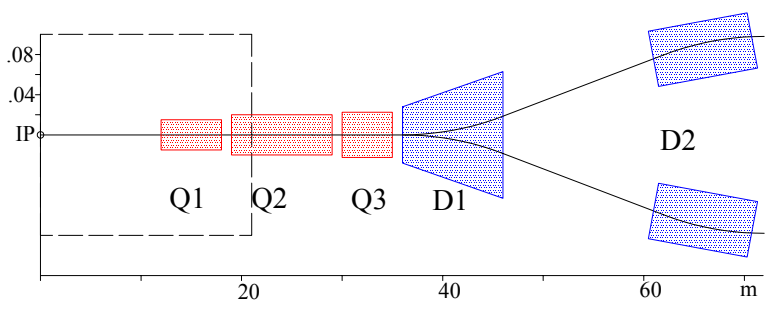

Figure 1: Layout of IR elements in optimized design. Scale is distorted to show location (horizontal), aperture (vertical). 
Table 1. Parameters of the IR elements, assuming $\mathrm{Nb}_{3} \mathrm{Sn}$ strand with $\mathrm{j}_{\mathrm{sc}}=3,000 \mathrm{~A} / \mathrm{mm}^{2}$ and $\mathrm{Cu}: \mathrm{SC}=1.5$.

$\begin{array}{lllll}\text { magnet } & \text { structure } & \begin{array}{l}\text { field/ } \\ \text { gradient }\end{array} & \begin{array}{l}\text { length } \\ (\mathrm{m})\end{array} & \begin{array}{l}\text { aperture } \\ (\mathrm{mm})\end{array} \\ \mathrm{Q}_{1} & \begin{array}{l}\text { Ironless, } \\ \text { round cable }\end{array} & 340 \mathrm{~T} / \mathrm{m} & 6.5 & 40 \\ \mathrm{Q}_{2} & \begin{array}{l}\text { Block-coil, } \\ \text { iron return }\end{array} & 450 \mathrm{~T} / \mathrm{m} & 10 & 50 \\ \mathrm{Q}_{3} & & 5 & 50 \\ \mathrm{D}_{1} & \begin{array}{l}\text { Levitated- } \\ \text { pole dipole }\end{array} & 9 \mathrm{~T} & 9 & 56 \times 120 \\ & & & \end{array}$
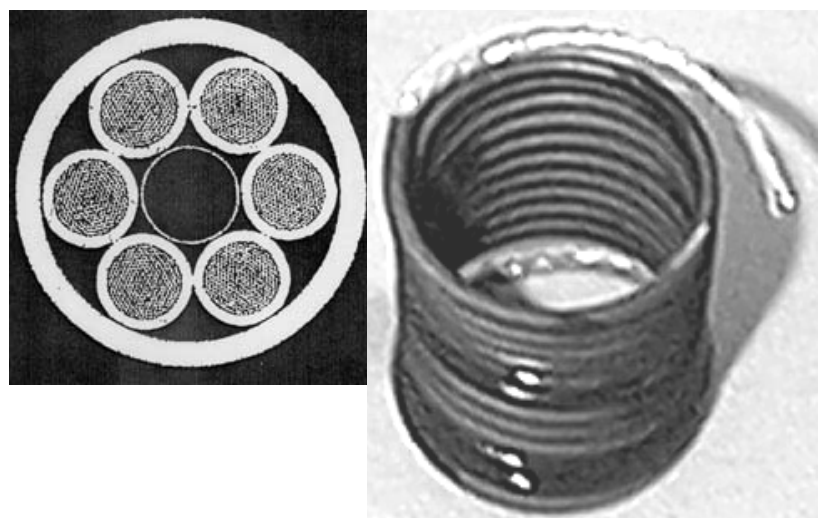

Figure 2. Structured cable: a) cross-section; b) test coil.

The cable is inserted within an Inconel 718 outer sheath (130 $\mu \mathrm{m}$ wall) and the sheath is compressed to preload the 6 strands against the center spring tube. Within the cable each strand is preloaded between the outer sheath, the inner tube, and the neighbor strands. The preload on the strand can be controlled by the amount of initial compression of the sheath so that it is $\sim$ twice the maximum Lorentz force upon a strand by the magnetic field at full excitation. The strands are thus immobilized within the cable without the requirement of impregnating the interior spaces of the cable.

The outer sheath provides a stress management structure within the overall winding. The Lorentz force on each cable element pushes primarily outwards from the axis of the quadrupole. The accumulation of Lorentz stress is conveyed through the outer sheaths of the cable elements, so that each strand is loaded only under the combination of the initial compression of the cable and the Lorentz force acting directly upon that strand.

The space between cables in the winding is vacuumimpregnated with epoxy, but with stress management within each cable element there is no need to impregnate the interior space (the black region in Figure 2a). This space is left open to provide a flow of superfluid helium through every cable element, thereby providing volumetric cooling to compensate for the volumetric heating delivered by particle losses. The sheath of each turn of cable is opened at the ends and parallel flow of superfluid helium is maintained in zones of $\sim$ uniform heat load. An operating temperature window $4.5 \rightarrow 6.0 \mathrm{~K}$ should be adequate to refrigerate a $300 \mathrm{~W}$ heat load.

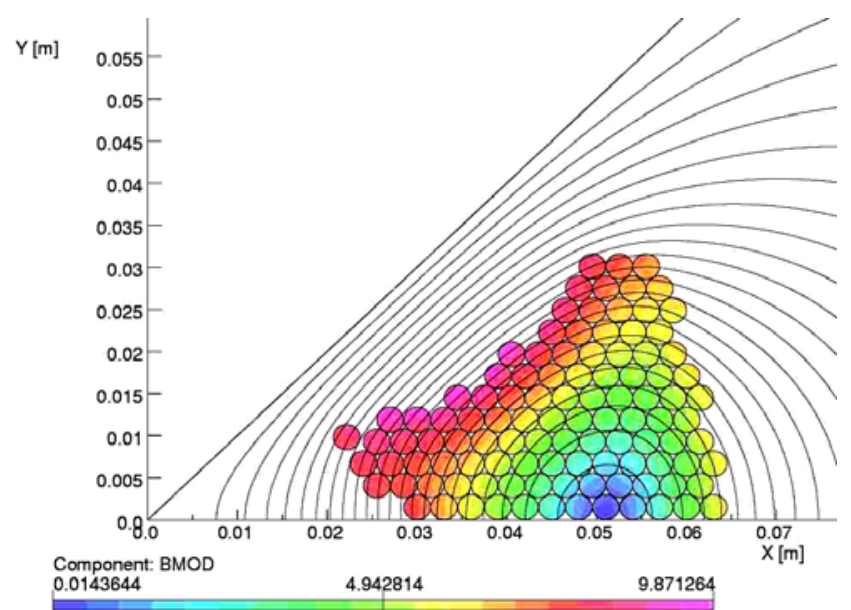

Figure 3. Octant cross-section of $\mathrm{Q}_{1}(340$ T/m @ 6 K). Field distribution within the coil is shown.

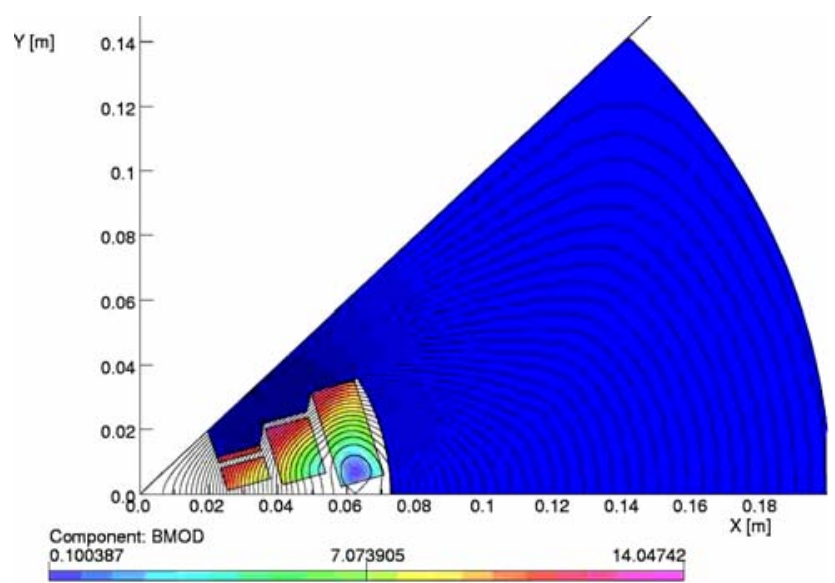

Figure 4. Octant cross-section of $\mathrm{Q}_{2}, \mathrm{Q}_{3}(450 \mathrm{~T} / \mathrm{m} @ 1.8 \mathrm{~K})$

The round cable geometry makes it possible to configure the quadrupole winding to take best advantage of the smaller aperture required for $\mathrm{Q}_{1}$. Figure $2 b$ shows a test coil of Bi-2212 structured cable that was formed on a bend radius of $12 \mathrm{~mm}$, equal to that required for the winding shown in Figure 3. When the cable is wrapped around the curvature, the outer sheath and spring core deform under strain, but the 6 strands inside retain their round cross section without deformation. The Bi-2212 test coil in Figure 2b was tested for critical current [3] and exhibited no degradation of $j_{c}$ from either cabling or coil bending. The placement of cables in the winding shown in Figure 3 uses the cable geometry to advantage to avoid regions with maximum field and at the same time produce uniform gradient in the bore.

Quadrupoles $\mathrm{Q}_{2}$ and $\mathrm{Q}_{3}$ are shadowed by $\mathrm{Q}_{1}$ and so do not receive a large heat load. We propose a block-coil design (with iron flux return) using conventional Rutherford cable as shown in Figure 4. Assuming refrigeration with superfluid helium, this design corresponds to a shortsample gradient of $450 \mathrm{~T} / \mathrm{m}$. 


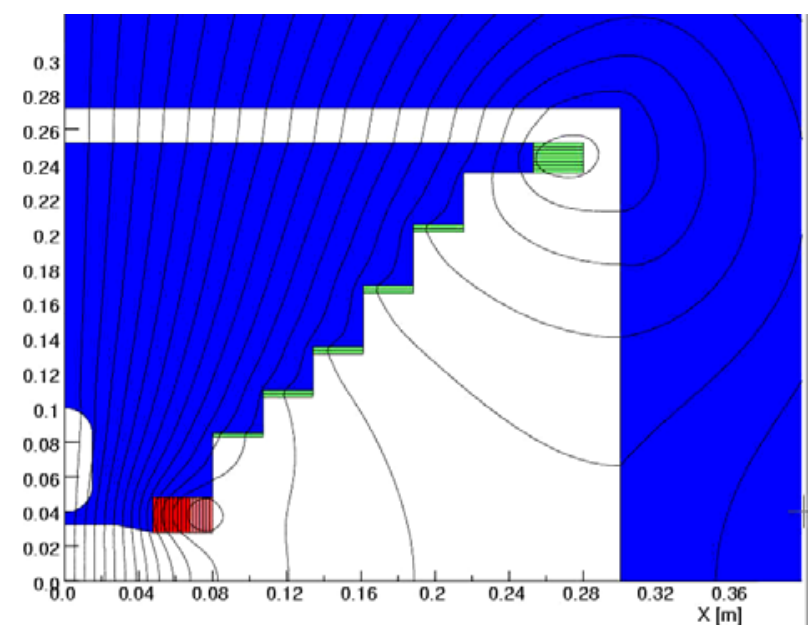

Figure 5. Levitated-pole dipole for $\mathrm{D}_{1} . \mathrm{Nb}_{3} \mathrm{Sn}$ winding in red, $\mathrm{NbTi}$ windings in green.

\section{LEVITATED-POLE DIPOLE}

Many of the most energetic high-energy particles from collisions at the IP are still traveling within the beam tube when they reach $D_{1}$ and most will be swept onto its side walls. For this reason the baseline IR design for LHC uses $\mathrm{Cu}$-coil dipoles for $\mathrm{D}_{1}$, and most upgrade design efforts have attempted to open a slot in the horizontal midplane [4]. The difficulties with open-slot designs is that it is difficult to support the windings on the poles, and the energy deposition in any case goes into the cold-iron flux return and presents a prodigious heat load.

We propose a somewhat different design in which the cold-iron poles of the dipole are supported within a warmiron flux return across a gap, as shown in Figure 5. The coil geometry and the separation gap can be designed such that the total Lorentz force on the assembly of the cold-iron pole piece and the coils is zero - we call this a levitated-pole dipole. The concept was first utilized by $\mathrm{T}$. Kawaguchi et al. [5] in the design of the sector magnets for a ring cyclotron. In this approach the radiation and heat from particles is deposited in room-temperature steel.

The magnetic field strength is limited by the requirement that the steel be unsaturated at the gap between pole tip and flux return. The design shown in Figure 5 corresponds to a central field of 9.0 Tesla (short sample limit for $\mathrm{Nb}_{3} \mathrm{Sn}$ winding). The pole is tapered at a $\sim 45^{\circ}$ angle to reduce the field in the pole steel from $\sim 9 \mathrm{~T}$ at the pole tip to $\sim 1.5 \mathrm{~T}$ at the gap. Figure 6 shows the calculated forces on the windings (red) and the cold-iron pole piece (green).

The design shown has been optimized for field quality; $b_{3} \sim 10^{-4} \mathrm{~cm}^{-2}$. Only the winding assembly just above the beam tube needs to be made using $\mathrm{Nb}_{3} \mathrm{Sn}$ superconductor. All of the windings along the staircase of the pole could be made of $\mathrm{NbTi}$ and operating at $4.2 \mathrm{~K}$.

Because the magnetic forces on the cold pole assembly cancel, it can be supported using low-heat-load tension supports as shown in Figure 7, so the overall cryogenic load should be modest.

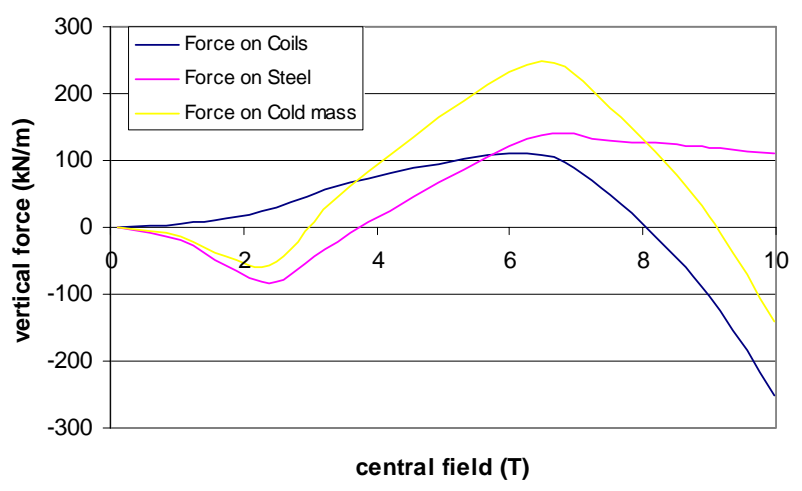

Figure 6. Vertical forces as a function of field: coils:(green, cold-iron pole (red), total (black).

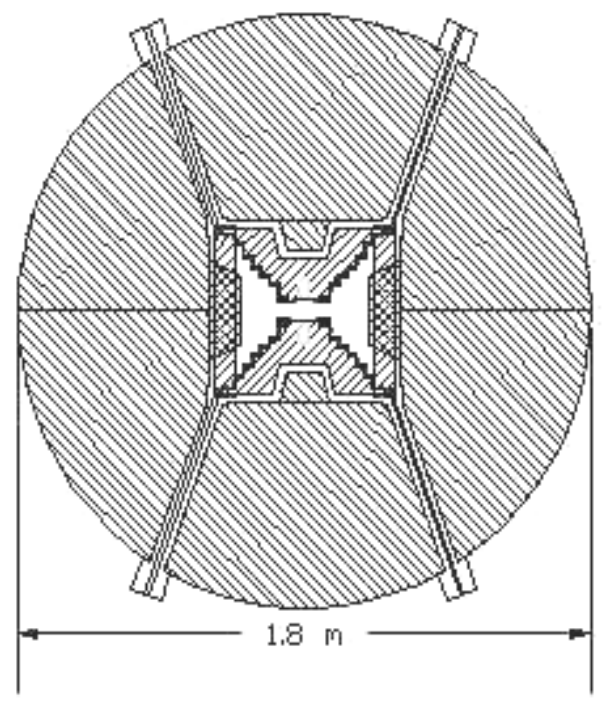

Figure 7. Support structure to mount cold-iron pole assembly within warm-iron flux return.

\section{IR OPTIMIZATION}

A mini- $\beta$ solution by which the focusing quadrupoles are pushed inside the experimental detector has already bbbbbbeen used in several colliders. In the LHC, this solution presents further specific advantages. The strength of the chromatic sextupoles in the arc corrections has been limited to maximize the packing factor. It is therefore important to avoid any significant increase of chromatic aberration arising from the IR insertions. The beams must cross at an angle in such a way that the beam separation at the long-range beam-beam interaction points reaches about $10 \sigma$ for nominal intensities and insertion length. Pushing the quadrupoles towards the IP linearly decreases the absolute beam separation and hence the required quadrupole aperture. Making the insertion more compact also reduces the number of long-range interactions which in turn allows a reduction of the crossing angle. These dependencies were coded into an optimization program implementing a crude insertion matching to explore the parameter space. The criteria for interesting solutions are the $\beta^{*}$, the quadrupole requirement, an 
evaluation of the first and second-order chromaticity, and the excitation of the first-order resonances driven by the systematic harmonics $b_{6}$ and $b_{10}$, relative to the nominal LHC.

The solutions with a free space of $13 \mathrm{~m}$ to $15 \mathrm{~m}$ to the $\mathrm{IP}$, gradients in the range of 300 to $390 \mathrm{~T} / \mathrm{m}$ with a maximum field of $13.5 \mathrm{~T}$ yields a $\beta^{*}$ of $0.25 \mathrm{~m}$, a $\beta_{\max }$ of 5.5 $\mathrm{km}$ and chromatic and geometric aberrations similar or lower than the nominal LHC. The $15 \mathrm{~m}$ free space solution even offers the potential to reduce $\beta^{*}$ to $0.15 \mathrm{~m}$ with a $\beta_{\max }$ of $11.5 \mathrm{~km}$, a linear chromaticity within the range of the lattice sextupoles, and all other aberrations similar to the nominal case. This latter result assumes a beam separation reduced to $7.5 \sigma$ thanks to a long-range beambeam compensation system [6]. These findings need now to be confirmed by an exact matching but provide a useful strategy in optimizing the LHC IRs.

\section{CONCLUSIONS}

New magnet technology is proposed to address the two most difficult design challenges in the optical elements of the intersection regions for LHC. Using these elements an optimized IR design is being investigated in which the quadrupole triplet begins $12-15 \mathrm{~m}$ from the IP and the beams are fully separated by $\sim 45 \mathrm{~m}$.

\section{REFERENCES}

[1] N.V. Mokhov et al., LHC Project Report 633 (2003).

[2] W. Scandale and T. Taylor, 'A design concept for the LHC insertion quadrupoles', Proc. PAC1991, p. 2260.

J. Strait et al., 'Towards a new LHC interaction region design for a luminosity upgrade', Proc. PAC2003.

F. Ruggiero et al., LHC Project Report 785 (2004)

[3] R. Soika et al., Physica C: Superconductivity 341-348, 2551-2554 (2000).

[4] R. Gupta et al., 'Open midplane dipole design for LHC IR upgrade', Proc. MT-18, Morioka, Japan (2003).

[5] T. Kawaguchi et al., "Design of the sector magnets for the Riken superconducting Ring Cyclotron", Proc. 15 Conf. on Cyclotrons, Caen, 14-19 June 1998.

[6] F. Zimmerman et al., 'Experiments on LHC longrange beam-beam compensation and crossing schemes at the CERN SPS in 2004', Proc. PAC05, Particle Accelerator Conf., Knoxville, TE, May 16-20, 2005. 